pixels at up to 120 frames per second, or even faster for smaller sections, which means that rapid physiological events can be visualized. According to Bernhard Zimmermann, head of product management at Zeiss, this shift from point- to line-scanning has minimal impact on resolution for most studies. "If you're looking at intracellular vesicles, you will hardly see a difference, he says.

\section{Adventures in the $n$th dimension}

These days a confocal microscope has to do more than simply track fluorophores. Multidimensional imaging is microscopy's new catchphrase, and the latest instruments gather information that goes beyond basic spatial orientation to provide time-lapse images and detailed fluorescence profiles.

The expansion in the range of available fluorescent labels means far more biological information can be colour-coded, and manufacturers have responded with advanced strategies for enhancing spectral resolution. For example, the LSM 510 META from Zeiss uses a 32-channel photomultiplier array behind an optical grating to generate full spectral signatures for each pixel; this is followed by further software-based separation. "You can even separate fluorescent emissions that peak at the same wavelength," says Zimmermann, "as long as the individual emission curves are different." Nikon offers a similar strategy with its $\mathrm{C} 1$ si confocal instrument, which can achieve spectral resolution as low as two nanometres.

Leica enhances confocal spectral precision with proprietary technologies that include the acousto-optical beam-splitter, which controls excitation illumination in a filter-free manner, and SP module, which uses a prism-based approach for precise spectral separation. These will be enhanced by its forthcoming introduction of white-light lasers, which provide coherent, continuous-spectrum illumination. ${ }^{\alpha}$ This will give us total freedom in excitation and detection for all available dyes," says Frank Olschewski, Leica's manager for confocal product development.

Fluorescence lifetime imaging (FLIM), which involves measuring the emission decay rate for an excited fluorophore, is also gaining interest for imaging. It can, for example, improve the quality of fluorescence resonance energy transfer (FRET) experiments. "When there is a FRET interaction, the donor lifetime gets shortened - this is an absolute, and it doesn't depend on the quantity or intensity," says John White of the Laboratory for Optical and Computational Instrumentation (LOCI) at the University of Wisconsin at Madison. ${ }^{\text {IIf }}$ you're doing other types of FRET measurements there are other reasons why you might get reduction in intensity or increased intensity, such as differential bleaching or com-

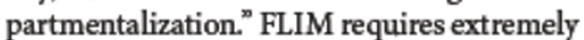
high-speed photon detectors; Becker \& Hickl of Berlin were an early leader in this regard, and Zeiss uses these detectors in its instruments. Leica also offers a FLIM attachment for its confocal instruments, which takes advantage of the SP detection system to provide 'spectral FLIM', with precise wavelength selection.

Standard fluorescence microscopes produce a focal spot that is ovoid rather than spherical. So although high-end instruments can produce images with $x-y$ resolution of up to 180 nanometres, the resolution is considerably poorer along the $z$ axis - around 500 to 800 nanometres - which reduces the quality of three-dimensional reconstructions.

One way round this uses two objective lenses, where the interference between the two optical wavefronts produces an effectively spherical focal spot. Stefan Hell and his colleagues at the Max Planck Institute for Biophysical Chemistry in Göttingen, Germany, demonstrated the effectiveness of this approach with their confocal $4 \mathrm{Pi}$ instrument, which can achieve a resolution of $100 \mathrm{~nm}$ in all directions. The original 4Pi design was relatively slow, barring its use in live specimens, but Hell and his team have since developed a multifocal, multiphoton version - MMM-4Pi - that scans samples with 64 foci, and images at a rate that makes highresolution three-dimensional imaging of live cells possible. A 'user-friendly' commercial version of Hell's 4Pisystem, the TCS $4 \mathrm{Pi}_{\mathrm{i}}$, is now available from Leica.

A related method, I5M, was developed by Mats Gustafsson of the University ofCalifornia, San Francisco. I5M also achieves axial resolution below $100 \mathrm{~nm}$, but is based on a wide-field configuration, with interference taking place over the entire field of view rather than at a point, and can be faster and potentially brighter than confocal 4Pi. Interference imaging produces

\title{
THINKING BIG, SEEING SMALL
}

Most op tical imaging techniques aresubject to the tyranny of the diffraction limit, where the optical properties of conventional objective lenses make it impossible to distinguish two objects separated byless than 180 nanometres in the focal plane.

But limits were made to be broken. In 1993, Stefan Hell of the Max Planck Institute for Biophysical Chemistry in Göttingen, Germany, developed the concept of stimulated emission depletion (STED). Fluorescent molecules are activated with a laser spot, which is then overlaid with a ring-shaped beam of lowenergy photons that shrinks the effective area of excitation to an extent determined by the brightness of the ring. This means that STED can generate almost arbitrarily small excitation areas. The instrument lives up to its promise, achievingresolution of well under 100 nanometres along all axes, and representing the first 'super resolution' technique to break the diffraction limit. This platform has evolved rapidly - Leica Microsystems of Wetzlar, Germany, plans to release a

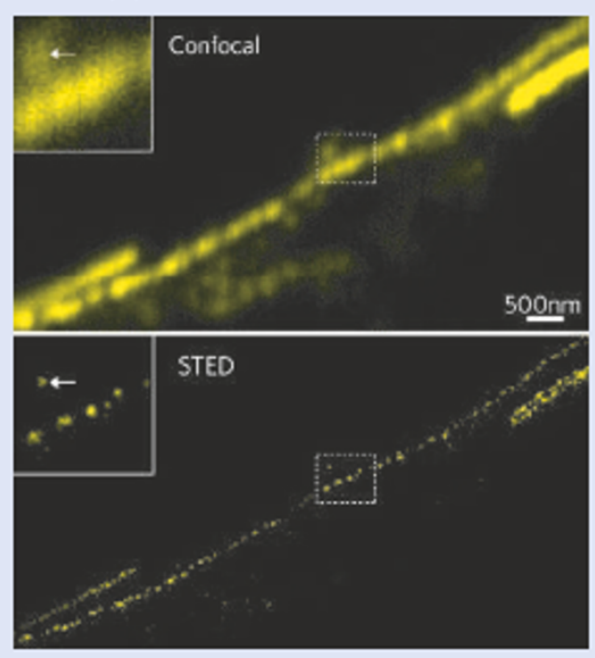

Stimulatedemission depletion provides improved resolution for immunostained neurofilaments compared with confocalmicroscopy. commercial instrument in 2007and Hell has also integrated STED with his confocal $4 \mathrm{Pi}$ instrument to push the resolution limit further.

"We'vegot a resolution of 15 to 30 nanometres in the focal plane," he says. Proof-of-concept studies suggest that STED should be suitable for live-cell imaging, although imaging rapid events with small focal volumes could prove tricky.

Fluorophore concentration is a major concern in super-resolution imaging. Mats Gustafsson, of the University of California, San Francisco, has demonstrated the use of patterned lightin a wide-field 'structured illumination' scheme that is effective for planar imaging at less than 50-nm resolution, but he feels that such techniques may be less ideal for smaller focal regions. "As your resolution volume shrinks, it holds a smaller and smaller number of molecules," he says, "and the individual stochastic response of each molecule becomes more app arent. Eventually, you reach a point where it is better to exploit, rather than fight, this independent molecular behaviour. This is precisely what Eric Betzig, for example, has done beautifully."

Betzig, of the Howard Hughes Medical Institute (HHMI) Janelia Farms campus in Virginia, cites the research of Hell and Gustafsson as fuelling his interest in superresolution. His efforts recently yielded a technique for singlemolecule imaging: photoactivated localization microscopy (PALM), which hedeveloped in 
artefactual 'lobes', which must be removed by deconvolution; these tend to be more prominent with $\mathrm{I} 5 \mathrm{M}$, and lobe removal is made easier by imaging with lenses that have a high numeri$\mathrm{cal}$ aperture. These are typically oil-immersion, and use of I5M is presently restricted to fixed specimens. Gustafsson's group has also developed a variant technique, I5S, which incorporates structured illumination to surpass the 'diffraction limit' in the image plane. Together, techniques such as I5S and confocal 4 Pi represent important early steps in 'super-resolution' microscopy, an increasingly vibrant area of research and technological development (see 'Thinking big, seeing small').

\section{Nonlinear thinking}

The real rising star of imaging is multiphoton microscopy (MPM), a nonlinear imaging method. In MPM, target molecules are stimulated by a pair (or more) of low-energy photons virtually simultaneously, providing sufficient energy in combination to induce excitation and photon emission. This approach uses long-wavelength, near-infrared pulsed lasers that can penetrate deep into tissues with heavy scattering properties and that are less likely to produce phototoxic side effects. Additionally, multiphoton excitation results in dramatically reduced background fluorescence. Price remains a major obstacle to adoption, as pulsed lasers are extremely expensive, but those who have tried the method generally walk away impressed. "I think it's the best technique in town for look- ing deep into solid tissue and figuring out the dynamics of what's going on," says White.

Zeiss currently controls the patent for femtosecond pulsed-laser MPM, and has made this technology available with its LSM 510 NLO. Several companies have also sublicensed this technology, and Olympus has made such an arrangement for its FV1000-MPE instrument, which was launched earlier this month. Leica also offers an MPM configuration for the TCS SP5, based on a separate patent that makes use of picosecond-pulse lasers.

Current MPM systems offer reasonable speed for live-cell work, but LaVision's TriMScope unit further improves imaging frame-rates with a multifocal approach that simultaneously scans samples with up to 64 foci, enabling the imaging of a $1004 \times 1002$ pixel field at 31 frames per second. TriMScope can also be adapted to perform FLIM or
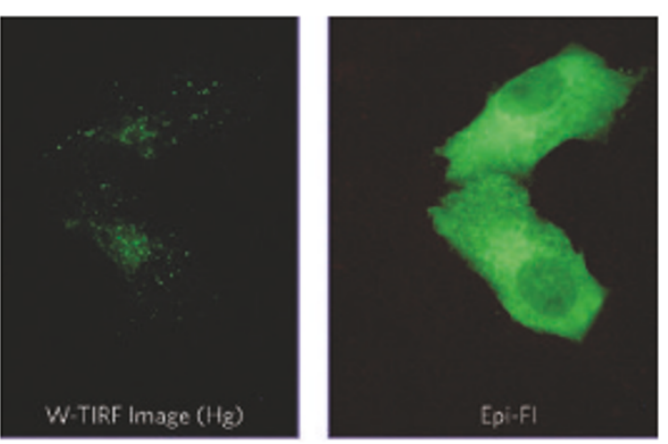

Nikon's white-light TIRF can be used to image individual clathrin-coated vesicles in the process of exocytosis. second-harmonic generation imaging, another nonlinear method gaining interest for its labelfree imaging capabilities.

\section{Scratching the surface}

Some of the most interesting biological events take place near the cell surface, and for imaging beauty that goes only membrane deep, total internal reflection fluorescence (TIRF) microscopy is a strong option. A sample on a glass coverslip is illuminated either through a prism or an objective lens of high numerical aperture. When light enters the coverslip at an angle greater than the critical angle, as determined by the difference between the refractive indices of the glass and the specimen, the incident beam does not enter the specimen, but instead produces an evanescent electromagnetic field. This field can excite fluorophores close to the surface (within about $200 \mathrm{~nm}$ ) of the coverslip, allowing non-destructive, live-cell imaging at the cell membrane with single-molecule resolution.

Most major microscope manufacturers now offer multi-wavelength TIRF modules for their inverted microscopes. These typically benefit from precise computer control of the angle and position of the excitation beam. Zeiss offers a compact, stand-alone option with its Laser TIRF system. TIRF instruments typically use oil-immersion lenses with a numerical aperture of 1.45 , an effective minimum for good imaging. Recently, Nikon broke this barrier with a set of Plan Apo 60x collaboration with Harald Hess of NuQuest Research in La Jolla, California. Subsets of individual photoactivatable fluorescent molecules are activated, and subsequentlybleached, within a sample through laser exposure, and then imaged by totalinternal reflection fluorescence (TIRF) microscopy. This process is repeated, and the resulting images aresuperimposed into a stack that is computationally resolved into a fluorescent molecule 'map' with resolution below $10 \mathrm{~nm}$.

The technique is not intended for live-cell work, but could offer a powerful super-resolution imaging tool for fixed cells. "There's good hope that it will be fairly easy to adapt for commercial TIRF," says Betzig. "I have some more grandiose ideas about how to do three-dimensional PALM, but the first order of business is to geta more usable instrument for cellbiologists."

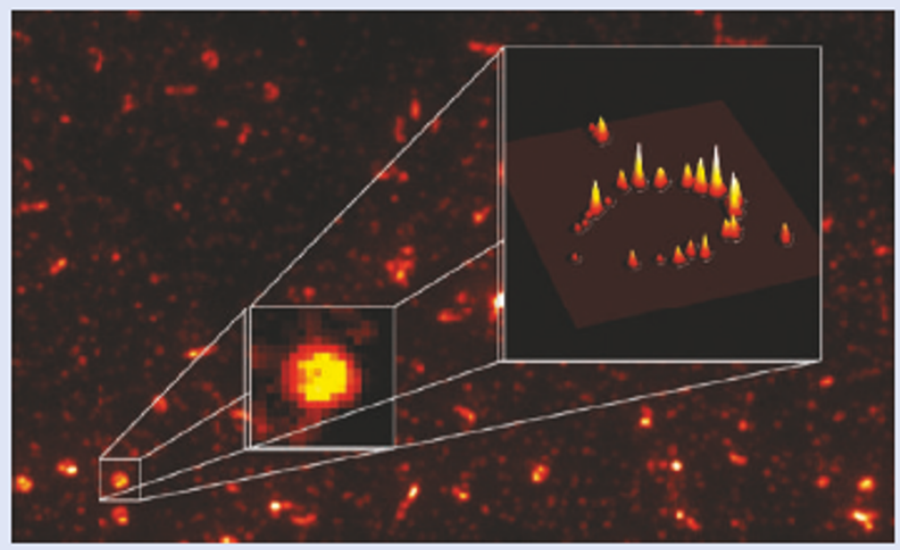

Imaging withstochastic optical reconstruction microscopy (inset) can reveal the detailed structure of $\operatorname{ReCA}$-DNA filaments.

HHMI investigator Xiaowei Zhuang, at Harvard University in Cambridge, Massachusetts, working with students Mark Bates and Michael Rust, recently described a similar technique, stochastic optical reconstruction microscopy (STORM). STORM emerged from the discovery by Zhuang's group that the commonly used Cy5 fluorop hore has photoswitchable properties. "It can be switched in a controlled way, back and forth hundreds of times, between light and dark states," says Zhuang. Like PALM, STORM involves cycles of selective excitation and imaging followed by computational reconstruction of the full image. As the fluorophores can beturned on and off, it is possible to do time-resolved imaging and faster imaging cycles. Resolution as low as 18 nanometres has been demonstrated, and Zhuang thinks this is just the beginning. Right now, her top priorities are exploring the potential for multicolour imaging, and improving imagingspeed. "If we can bring STORM toone-second resolution, that's going to open up a big door and enablemany different things," shesays.

Surveying the field, $\mathrm{Hell}$ views these various approaches as complementary tools that advance a common goal - forcing researchers to re-examine what is possible in imaging. "I think breaking the diffraction barrier is a fundamental step forward," he says. "This is an idea whose time has been coming for a long timenow." 